\title{
Top physics prospects in ATLAS
}

\section{From early data to precision measurements}

\author{
Arnaud Lucotte ${ }^{1}$ \\ LPSC-IN2P3 - 53, Av. des martyrs, 38000 Grenoble, France
}

Received: date / Revised version: date

\begin{abstract}
Top Physics aspects are reviewed. A particular emphasis is put on the precision measurements of the top mass, top polarization and on the single-top cross-section measurments during the low luminosity period of the LHC data taking.
\end{abstract}

\section{Introduction}

The discovery of the Top quark at Fermilab's collider in 1995 by the CDF and DØ collaborations suggested a confirmation of the three generation quark family as predicted by the Standard Model of particle physics. Since then, determinations of top quark properties, its mass, spin, charge and couplings to fermions or bosons have been investigated. But the precision for most of these measurements is still statistically limited and will most presumably still be at the end of a $2 \mathrm{fb}^{-1}$ TeVatron run.

With more than 8 millions of top pair and more than 2 millions of single top events produced every year at low luminosity, the LHC era will open a new opportunity for top quark physics. One of the first goals will be a determination of the top quark mass at the $1 \%$ level. This determination constitutes a crucial test of the electro-weak sector and put stringent constraints onto its symmetry breaking mechanism, either in the Standard Model (SM) or in a supersymmetric framework (MSSM). The top quark spin properties, through $\mathrm{W}$ polarization and top spin correlation measurements at a precision better than $5 \%$ level, will also lead to a deep insight of the nature of the top quark couplings to fermions and to the mechanisms (SM or not) responsible for its production. Finally, a precise determination of the (electro-weak) single-top production cross-sections at a few percent precision level also constitutes a stringent test of the SM. These measurements offer a direct access to $V_{\text {tb }}$ at the few percent level, as well as stringent tests of any departure to SM physics with a sensitivity to anomalous couplings. Single-top analyses can also be a direct way of evidence for an extra charged Higgs bosons.

\section{Top quark mass measurement}

Fig. 1 displays the present experimental values taken by the top and $\mathrm{W}$ boson masses compared to their predictions in the SM or in an unconstrained SUSY models [1].
In the SM, the indirect precision measurements of the electro-weak sector ( $\mathrm{Z}$ resonance, etc..) tend to favour the presence of a light neutral Higgs below $295 \mathrm{GeV} / \mathrm{c}^{2}$ at $95 \%$ CL [2].

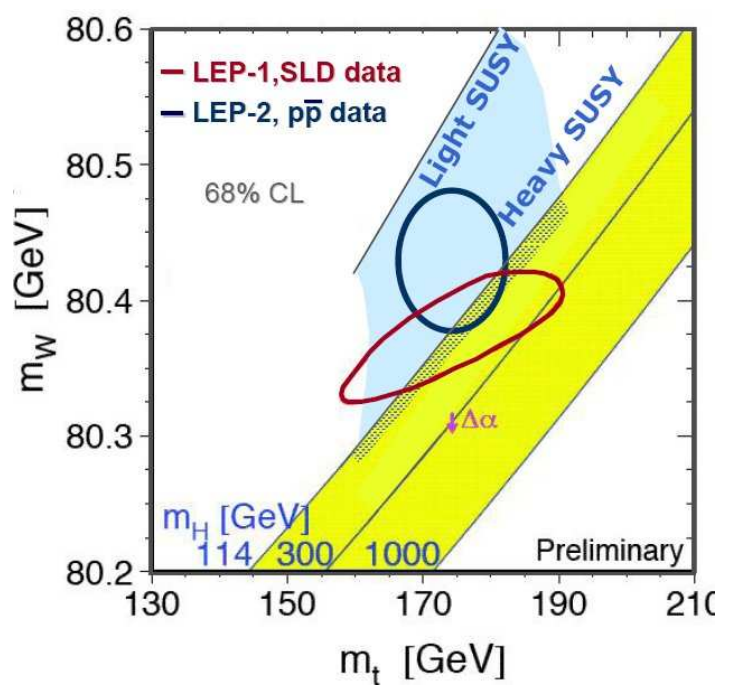

Fig. 1. Constraints from precise determination of $m_{W}$ and $m_{t}$ on the electro-weak sector of the $S M$ (yellow band) and MSSM framework (blue band)

In the MSSM, the mass of lightest Higgs is predicted and must lie below $135 \mathrm{GeV} / \mathrm{c}^{2}$. In both cases, given the level of the present precision in $\Delta \mathrm{m}_{\mathrm{t}}$ and $\Delta \mathrm{m}_{\mathrm{W}}$, no particular framework appears yet as the preferred one. The main source of uncertainty in the global fitted Higgs mass actually is the precision of $\mathrm{m}_{\mathrm{t}}$ with $\Delta \mathrm{m}_{\mathrm{W}} \simeq 0.7 \% \Delta \mathrm{m}_{\mathrm{t}}$, making a $\Delta \mathrm{m}_{\mathrm{t}}=1 \mathrm{GeV} / \mathrm{c}^{2}$ the target for the LHC.

At the LHC, only top pair events have been used so far for the determination of the top mass. Originating from gluon fusion (90\%) and quark anihilation (10\%), the corresponding cross-section has been computed up to the 
Next To Leading Order (NLO) of corrections resulting in $\sigma_{\mathrm{t} \overline{\mathrm{t}}}=835 \mathrm{pb}$ [3] for $\mathrm{m}_{\mathrm{t}}=175 \mathrm{GeV} / \mathrm{c}^{2}$ with a $10 \%$ uncertainty. Within the SM, the top decays almost exclusively into a $\mathrm{W}$ boson and a b quark. Signatures of top pair events thus depend exclusively upon the $\mathrm{W}$ boson decays, and are splitted into three samples: a 'lepton+jets' sample, where one $\mathrm{W}$ decays hadronically while the other decays into a charged lepton (electron and muon) and a neutrino, with a branching ratio (BR) of about 30\%; a 'dilepton' sample, where both W's decay into a lepton and a neutrino with a branching ratio of $5 \%$ and a 'full hadronic' channel where all $\mathrm{W}$ bosons decay into hadrons, which occurs in about $44 \%$ of the time.

\subsection{Top mass in the 'lepton+jets' channel}

A detailed report of this analysis may be found in Ref. [4]. The preselection of such events requires an isolated high $\mathrm{p}_{\mathrm{T}}$ lepton, a high missing energy due to the undetected neutrino and at least four jets with $\mathrm{p}_{\mathrm{T}}$ above $40 \mathrm{GeV} / \mathrm{c}$. Among those four jets, one jet at least must be tagged as a $\mathrm{b}$ jet. The signal efficiency is about $3.5 \%$ resulting in about 87,000 events for $\mathcal{L}=10 \mathrm{fb}^{-1}$ for the $2 \mathrm{~b}$-tag sample, while physics backgrounds, formed by the QCD b $\bar{b}, W / Z+$ jets and di-boson productions add up to a total below 2,000 events. For the 1 btag sample, the efficiency is about $11 \%$ with a physical background about 10 times larger. In both cases, the main background to the mass determination is composed by the jet-pairing combinatorial of top pair events themselves. Selected events are then separated into two classes according to the number of b-tagged jets, a single b-tag and a 2 b-tag samples.

The mass determination makes use of the top quark decaying hadronically $\mathrm{t} \rightarrow \mathrm{Wb} \rightarrow$ jjb by reconstructing the three jet invariant mass $\mathrm{M}_{\mathrm{jjb}}$. The $\mathrm{W}$ boson is first reconstructed using the invariant mass formed by all two-jet combinations among non b-tagged jets, keeping the solution with the closest value to the $\mathrm{W}$ mass. This approach leads to an overall W purity of $66 \%$ (55\%) in the '2-btag' ('1-btag') sample for a right combination contained in the $\left|\mathrm{m}_{\mathrm{jj}}-\mathrm{m}_{\mathrm{W}}\right|<20 \mathrm{GeV} / \mathrm{c}^{2}$ window, and corresponds to an overall efficiency of $3.7 \%$. A b-jet must then be associated to the reconstructed W. In the '1-btag' sample, the association is performed if the $\mathrm{b}$ jet is closer to the $\mathrm{W}$ than to the isolated lepton. For '2-btag' events, the b-jet leading to the highest top transverse momentum is chosen. An overall efficiency of $1.2 \%(2.5 \%)$ is achieved for a corresponding purity of $69 \%$ (65\%) for events such that $\left|\mathrm{m}_{l \nu \mathrm{b}}-\mathrm{m}_{\mathrm{t}}\right|<35 \mathrm{GeV} / \mathrm{c}^{2}$. The event yields are about $30 \mathrm{~K}$ expected events for a $12 \mathrm{GeV} / \mathrm{c}^{2}$ resolution, resulting to a statistical uncertainty below $0.1 \mathrm{GeV} / \mathrm{c}^{2}$ [4].

A mismeasurement of $1 \%$ of jet energy induces a top mass shift of $1.6 \mathrm{GeV} / \mathrm{c}^{2}$. Similarly, a mismeasurement of $1 \%$ in the cosine of the opening angle for $\mathrm{W}$ jets or between the b-jet and the $\mathrm{W}$ direction results in a mass shift of $1.2 \mathrm{GeV} / \mathrm{c}^{2}$. An in situ calibration of direction and energy of light jet is then performed using a purified sample of $\mathrm{W} \rightarrow \mathrm{jj}$ events, by keeping only jets with $\left|\mathrm{m}_{\mathrm{jj}}-\mathrm{m}_{\mathrm{W}}\right| \leq 15 \mathrm{GeV} / \mathrm{c}^{2}$. This sample is used to correct for the energy scale bias as well as for mis-estimate of the jet direction. The proper use of this technique could result in a determination of the absolute energy scale at the $1 \%$ level [4]. This determination will be checked with an external calibration based on the use of $\mathrm{Z}+$ jets events, that is shown to systematically underestimate the energy sharing of $\mathrm{W} \rightarrow$ jj jets.

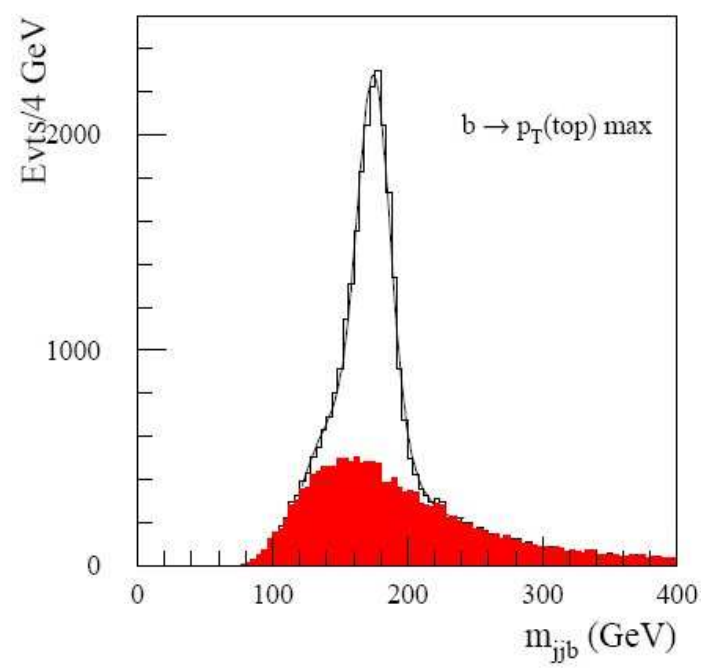

Fig. 2. Invariant mass reconstructed in the "2-btag" sample [1]

The main challenge to the top mass determination is the control of the systematic biases. The dominant source of uncertainty comes from the knowledge of the jet energy scale. For b-jet, a $1 \%$ mismeasurement results in a shift of $0.7 \mathrm{GeV} / \mathrm{c}^{2}$ in the top mass, that grows linearly with the miscalibration factor. Regarding light jets, a $1 \%$ mismeasurement induces a $0.2 \mathrm{GeV} / \mathrm{c}^{2}$ mass shift. In this case, the use of the in situ calibration will be a determinant factor. Initial state radiation directly influences the number of reconstructed jets in the event, thus leading to inefficiencies in the light jet association to the W. Final state radiations affect the jet reconstruction through gluon radiations which lead generally to an understimated jet energy. This effect can result in a mis-estimate of the selection efficiency due to jets cut by the preselection threshold. Such effects also show a dependence to the jet reconstruction algorithm parameters, depending crucially on the cone size. The corresponding quoted systematics represents $20 \%$ of the mass shift due to the addition of the ISR or FSR in the Monte Carlo, which is conservative with respect to the uncertainty in the strong coupling constant $\alpha_{\mathrm{s}}$.

A way to reduce the systematics is to use the leptonic decay of the other top quark as a constraint. A kinematic fit is applied to the entire t $\bar{t}$ event by reconstructing both the leptonic and hadronic $\mathrm{W}$ bosons and by requiring the leptonic top mass to be equal to the hadronic one. It is shown that the formed $\chi^{2}$ can be used to reduce the contribution from badly reconstructed jets due to FSR effects. This method thus directly shows up in the systematics as- 
Table 1. Sources of uncertainty and size of the effects on the top mass determination. Numbers into parenthesis correspond to the leptonic kinematic fit constraint [4]

\begin{tabular}{lccc}
\hline Sources of & \multicolumn{3}{c}{$\delta \mathrm{m}_{\mathrm{t}}$ in $\mathrm{GeV} / \mathrm{c}^{2}$} \\
uncertainty & 'lepton+jets' & 'dilepton' & 'full hadron' \\
\hline jet energy scale & 0.2 & - & 0.8 \\
b-jet energy scale & $0.7 \times \Delta \%$ & $0.7 \times \Delta \%$ & $0.7 \times \Delta \%$ \\
ISR & 0.1 & 0.6 & 2.8 \\
FSR & $1.0(0.5)$ & 0.6 & 2.8 \\
b-quark frag. & 0.1 & 0.7 & 0.3 \\
combinatorial & 0.1 & - & 0.4 \\
Pdf's & - & 1.2 & - \\
\hline
\end{tabular}

sociated to the FSR effects, by limiting the effect below $0.5 \mathrm{GeV} / \mathrm{c}^{2}$. Providing a miscalibration factor of $1 \%$ in the b-jet and light jet energy scales, a total systematic uncertainty around $1 \mathrm{GeV} / \mathrm{c}^{2}$ seems achievable.

\subsection{Top mass in the 'dilepton' and 'full-hadronic' channels}

The 'dilepton' analysis is presented in details in Ref. [4][5]. Trigger and selection are based upon the detection of two isolated high $\mathrm{p}_{\mathrm{T}}$ leptons of opposite signs, a high transverse missing energy due to the presence of two neutrinos in the final state, and at least two high $\mathrm{p}_{\mathrm{T}}$ jets, among which one or two at least has to be b-tagged. About $80 \mathrm{~K}$ events are expected to be selected in $10 \mathrm{fb}^{-1}$ with a ratio $\mathrm{S} / \mathrm{B} \approx 10$. Each $\mathrm{t} \overline{\mathrm{t}}$ event is then fully reconstructed by solving a system of 6 equations and 6 unknowns (3 components of neutrino momenta) based upon the conservation of the overall transverse momentum of the $t \bar{t}$ system, the mass constraint on the lepton+neutrino coming from the $\mathrm{W}$, as well as top mass constraints on to the lepton, neutrino and $\mathrm{b}$ jet. The complete kinematic reconstruction can be performed with an efficiency above $97 \%$ and with the right solutions in $73 \%$ events.

For the top mass determination, the reconstruction algorithm is fed with different top mass inputs. For each solution a weight is then attributed according to the fit comparing the event topology and kinematics with the $\mathrm{MC}$ expectations. The top mass is then defined as the preferred value on an event by event basis. The final top mass is obtained using the full sample by fitting the distribution of all event weights.

Main systematics comes from the miscalibration effect of b-jets, which accounts for $0.6 \mathrm{GeV} / \mathrm{c}^{2}$ in the mass, as well as ISR/FSR modelling. Variation of b-quark fragmentation parameters result to an error of $0.6 \mathrm{GeV} / \mathrm{c}^{2}$. The new source of uncertainty comes from the high dependence to the MC simulation used to attribute the weight. This shows up in the parton distribution function contribution. An overall systematics of $1.6 \mathrm{GeV} / \mathrm{c}^{2}$ seems achievable.

The full hadronic channel is most challenging given the high level of jet background. Based on the selection of at least 6 central high $\mathrm{p}_{\mathrm{T}}$ jets with 2-btagged jets, the analysis makes use of kinematical, topological and event shape variables to discriminate the signal from the backgrounds. A constrained kinematic fit to both $\mathrm{W}$ bosons and to top mass distributions is performed using only events with high $\mathrm{p}_{\mathrm{T}}$ reconstructed top candidates. A mass resolution of $13 \mathrm{GeV} / \mathrm{c}^{2}$ is achieved for about 3,300 events remaining with a ratio S/Bapprox 18/1. Systematics are completely dominated by the FSR modelling, b-jet and light jet energy scale. An overall uncertainty of $3.1 \mathrm{GeV} / \mathrm{c}^{2}$ seems achievable.

For all the present analyses, the main source of systematics comes from the b-jet energy scale. This result lead to the developpment of an alternative analysis. This approach is based on the identification of the $\mathrm{J} / \Psi$ originating from the b-quark decay and uses the linear correlation in $\mathrm{m}_{\mathrm{t}}$ of the reconstructed invariant mass $\mathrm{M}(\mathrm{l}, \mathrm{J} / \Psi)[6]$. While this approach is not affected by the b-jet energy scale uncertainty, it however is characterized by a small $\mathrm{BR} \approx 5 \times 10^{-5}$ and requires $100 \mathrm{fb}^{-1}$ at high luminosity to achieve a precision around $1 \mathrm{GeV} / \mathrm{c}^{2}$.

\section{$3 \mathrm{~W}$ and top quark polarization in $t \bar{t}$ events}

Because of its high mass, the top quark decays before it hadronizes or its spin flips, thus leaving an inprint of its spin on its angular decay distributions [7]. This feature constitutes a unique opportunity to measure quark spin properties. The measurements of the $\mathrm{W}$ boson and top polarization constitute a test of both the top production and $\mathrm{W}$ decay with the same initial sample.

A detailed report of the analyses conducted in ATLAS may be found in Ref. [8]. Top pairs are selected similarly as they are in the top mass analyses, ending up with 85,000 signal events in the 'lepton+jets' sample and 21,000 events in the 'di-lepton' sample [9]. To enhance the spin correlation effects, selected events are required to have a reconstructed invariant mass $\mathrm{M}_{\mathrm{t} \overline{\mathrm{t}}}$ below $550 \mathrm{GeV} / \mathrm{c}^{2}$.

\subsection{W polarization measurement}

W bosons decay of top quarks are produced with a longitudinal, left-handed or a right-handed polarization. In t $\bar{t}$ events, $\mathrm{W}$ bosons are mainly produced longitudinally with the corresponding probabilities $\mathrm{F}_{0}=0.695, \mathrm{~F}_{\mathrm{L}}=0.304$ and $\mathrm{F}_{\mathrm{R}}=0.001$ for a $\mathrm{W}^{+}[10]$. Thus, any deviation of $\mathrm{F}_{0}$ from the SM value would pintpoint an inconsistency in the Higgs mechanism, responsible for the longitudinal degree of freedom of the massive bosons. Any deviation seen in $\mathrm{F}_{\mathrm{L}}$ or $\mathrm{F}_{\mathrm{R}}$ would be a sign of additional $(\mathrm{V}+\mathrm{A})$ admixture as predicted in the $\mathrm{SU}(2)_{\mathrm{L}} \times \mathrm{SU}(2)_{\mathrm{R}} \times \mathrm{U}(1)$ extensions of the SM [11].

\subsubsection{Method}

The W-polarisation is measured from the angular distribution of its decay products : the charged lepton from a left-handed (right-handed) $\mathrm{W}^{+}$tend to be emitted in the opposite (same) $\mathrm{W}^{+}$direction, leading to a softer (harder) 
$\mathrm{p}_{\mathrm{T}}$ spectrum than the lepton from a longitudinal W. As the knowledge of the isospin of the produced fermion is necessary to assess the helicity of the initial decaying W, charged leptons are the best candidates or 'spin analyzer'.

Both 'di-leptonic' and 'lepton+jets' samples have been used. The polarization is assessed via the measurement of $\Psi$ defined as the angle between the lepton direction in the $\mathrm{W}$ rest frame and the $\mathrm{W}$ direction in the top quark rest frame:

$$
\begin{gathered}
\frac{1}{\mathrm{~N}} \frac{\mathrm{dN}}{\mathrm{d} \cos \Psi}= \\
\frac{3}{2}\left[\mathrm{~F}_{0}\left(\frac{\sin \Psi}{\sqrt{2}}\right)^{2}+\mathrm{F}_{\mathrm{L}}\left(\frac{1-\cos \Psi}{2}\right)^{2}+\mathrm{F}_{\mathrm{R}}\left(\frac{1+\cos \Psi}{2}\right)^{2}\right]
\end{gathered}
$$

As both rest frames are used in the analysis, the event topology has to be fully reconstructed, which makes the 'lepton+jets' sample the best choice for such analysis. In the 'di-lepton' sample, because of the presence of two neutrinos, the $\Psi$ angle is reconstructed using the following relation [12]:

$$
\cos \Psi \approx \frac{2 \mathrm{M}_{\mathrm{lb}}^{2}}{\mathrm{~m}_{\mathrm{t}}^{2}-\mathrm{m}_{\mathrm{W}}^{2}}-1
$$

where $\mathrm{m}_{\mathrm{t}}$ and $\mathrm{m}_{\mathrm{W}}$ are set to $175 \mathrm{GeV} / \mathrm{c}^{2}$ and $80.41 \mathrm{GeV} / \mathrm{c}^{2}$.

The selection requirements affect both the reconstructed $\mathrm{p}_{\mathrm{T}}$ and the angular distributions of the physics objects. A procedure has been defined to recover the original shape of the $\cos \Psi$ via the use of a weighting function applied on an event by event basis. This correction function results from the fitted ratio of the normalized $\cos \Psi$ distributions at the reconstructed level over the generated level. It is computed on an independent data sample and then applied event by event on the analysis sample.

The search for non-SM contributions lead to the developpement of a (quasi-) independent correction function that is formed iteratively, by starting from the SM function and re-injecting the non-zero fitted value of $F_{R}$ as input to the new function. It is shown that a few iterations are enough to reach convergence.

\subsubsection{Results and sensitivity to new physics}

Table 2 reports the performance expected in the SM framework for an integrated luminosity of $10 \mathrm{fb}^{-1}$. Combining 'lepton+jets' and 'di-lepton' analyses, $\mathrm{F}_{\mathrm{L}}$ and $\mathrm{F}_{\mathrm{R}}$ are determined at a few percent precision level. The results are 3 to 5 times better than the statistical sensitivity expected at the TeVatron with $2 \mathrm{fb}^{-1}$ [13].

Table 2. W polarization results with $10 \mathrm{fb}^{-1}$ at $10^{33} \mathrm{~cm}^{-2} \mathrm{~s}^{-1}$ Central values are those predicted by the Standard Model at LO

\begin{tabular}{ccc}
\hline & LHC $\left(10 \mathrm{fb}^{-1}\right)$ & TeVatron $\left(2 \mathrm{fb}^{-1}\right)$ \\
\hline $\mathrm{F}_{\mathrm{L}}$ & $0.303 \pm 0.003_{\text {stat }} \pm 0.024_{\text {syst }}$ & \\
$\mathrm{F}_{0}$ & $0.697 \pm 0.004_{\text {stat }} \pm 0.015_{\text {syst }}$ & $\pm 0.09_{\text {stat }}$ \\
$\mathrm{F}_{\mathrm{R}}$ & $0.000 \pm 0.003_{\text {stat }} \pm 0.012_{\text {syst }}$ & $\pm 0.03_{\text {stat }}$ \\
\hline
\end{tabular}

Measurements are largely dominated by the systematic uncertainties. At the generation level, the main systematics originate from the scale used for the parton generation, the uncertainty in the generated top mass and the choice of the pdf's. Biases due to the event simulation and reconstruction come from the effects of ISR/FSR on the angles and energy reconstruction, the uncertainty on the top mass knowledge as well as the b-jet energy scale that directly affects the determination of $\cos \Psi$. Uncertainty in the determination of the background and pile-up effects have also been taken into account.
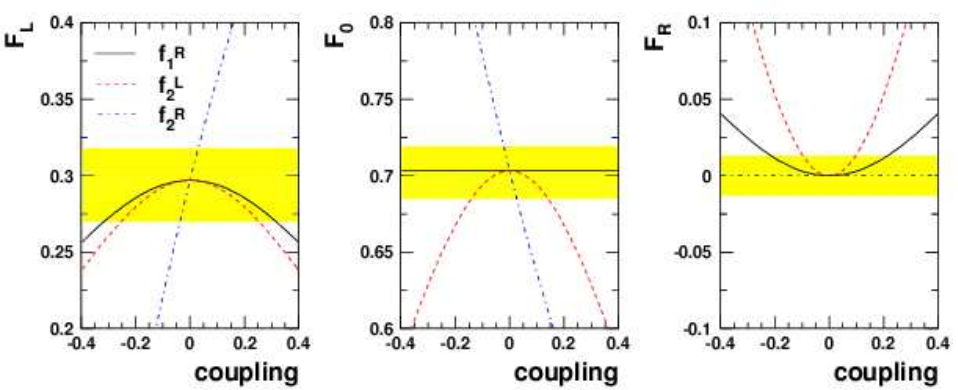

Fig. 3. Sensitivity to anomalous couplings via the measurement of $\mathrm{F}_{\mathrm{L}}, \mathrm{F}_{0}$ and $\mathrm{F}_{\mathrm{R}}$ with $10 \mathrm{fb}^{-1}$. Grey bands correspond to $1 \sigma$ uncertainty

In a more general effective CP-conserving lagrangian, the Wtb interaction can be parametrized using $\mathrm{f}_{1}^{\mathrm{L}}, \mathrm{f}_{1}^{\mathrm{R}}$ as vectorlike couplings and $\mathrm{f}_{2}^{\mathrm{L}}, \mathrm{f}_{2}^{\mathrm{R}}$ as tensor-like couplings. In the $\mathrm{SM} \mathrm{f}_{1}^{\mathrm{L}}=\mathrm{V}_{\text {tb }}$ while $\mathrm{f}_{1}^{\mathrm{R}}=\mathrm{f}_{2}^{\mathrm{L}}=\mathrm{f}_{2}^{\mathrm{R}}=0$. The sensitivity to those quantities are shown on Fig. 3 together with the expected precision on $\mathrm{F}_{\mathrm{L}}, \mathrm{F}_{0}$ and $\mathrm{F}_{\mathrm{R}}$.

\section{2 top quark polarization measurement}

In the top pair production, top quarks are not polarized. However, the top and anti-top spins are correlated due to their production mechanism: the $\mathrm{q} \overline{\mathrm{q}}$ anihilation generates a ${ }^{3} \mathrm{~S}_{1}$ state resulting in aligned top and anti-top spins directions, while the gluon fusion produces a ${ }^{1} \mathrm{~S}_{0}$ final state leading to opposite direction spins. In the helicity basis, the following observable is used :

$$
\mathcal{A}=\frac{\sigma\left(\mathrm{t}_{\mathrm{L}} \overline{\mathrm{t}}_{\mathrm{L}}\right)+\sigma\left(\mathrm{t}_{\mathrm{R}} \overline{\mathrm{t}}_{\mathrm{R}}\right)-\sigma\left(\mathrm{t}_{\mathrm{L}} \overline{\mathrm{t}}_{\mathrm{R}}\right)-\sigma\left(\mathrm{t}_{\mathrm{R}} \overline{\mathrm{t}}_{\mathrm{L}}\right)}{\sigma\left(\mathrm{t}_{\mathrm{L}} \overline{\mathrm{t}}_{\mathrm{L}}\right)+\sigma\left(\mathrm{t}_{\mathrm{R}} \overline{\mathrm{t}}_{\mathrm{R}}\right)+\sigma\left(\mathrm{t}_{\mathrm{L}} \overline{\mathrm{t}}_{\mathrm{R}}\right)+\sigma\left(\mathrm{t}_{\mathrm{R}} \overline{\mathrm{t}}_{\mathrm{L}}\right)}
$$

$\mathcal{A}$ can be written as function of the measured angular distributions of $\theta_{1}$ and $\theta_{2}$, where $\theta_{1}\left(\theta_{2}\right)$ of the $t(\overline{\mathrm{t}})$ spin analyzer in the $t(\overline{\mathrm{t}})$ rest frame and the $\mathrm{t}(\overline{\mathrm{t}})$ direction in the $t \bar{t}$ center of mass of the system, are used to estimate the $t \overline{\mathrm{t}}$ correlation. Another observable $\mathcal{A}_{\mathcal{D}}$ defined in [8]

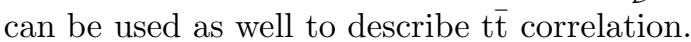




\subsubsection{Results}

A weighting function is defined iteratively following the procedure used for the $\mathrm{W}$ polarization measurements. The correction function is computed this time by fitting the $\cos \theta_{1} \cos \theta_{2}$ distributions on an independent sample. The sensitivity in the 'lepton+jets' channel is, again, driven by the systematic uncertainties. This is no longer true in the 'di-lepton' analysis where statistical error is similar to the systematic one. The sources of systematics are similar to the one listed in the previous section.

A precision of $6.5 \%$ in $\mathcal{A}$ and below $5 \%$ in $\mathcal{A}_{\mathcal{D}}$ can be achieved in the SM framework. These results can be compared with the TeVatron $40 \%$ precision (stat.) expected with a luminosity of $2 \mathrm{fb}^{-1}$. Any deviation from the SM predictions can sign the presence of new heavy resonances in the $t \bar{t}$ production of spin- 0 particule $(\mathrm{H} \rightarrow \mathrm{t} \overline{\mathrm{t}})$ or spin-2 particule (Kaluza-Klein gravitons). It can also probe presence of technicolor or topcolor theories.

\section{Single-top cross-section measurement}

Although non-dominant, the single-top production represents a third of the total top quark pair production. If a $5 \sigma$-evidence of single-top events at the Fermilab p $\bar{p}$ collider seems to be achievable with $2-4 \mathrm{fb}^{-1}$, precise measurements will only be possible at the LHC. The measurement of all three contributions to the total cross-section will provide a valuable test of the electro-weak top production, which in turn, will allow the first direct determination of $\mathrm{V}_{\mathrm{tb}}$ at the $1 \%$ level of precision. They also constitute a powerful probe for new physics, being sensitive to additional contributions from an extra charged Higgs boson as predicted by 2HDM models.

\subsection{Event selection}

In the SM the electroweak single-top production is due to three different mechanisms: the W-boson gluon fusion mode $\mathrm{Wg}$ or t-channel contribution $(\mathrm{Wg})$; the associated production of a top quark and a $\mathrm{W}(\mathrm{W}+\mathrm{t})$; and the schannel coming from the exchange of an off-shell mass $\mathrm{W}^{*}$. For leptonic $\mathrm{W}$ decays, the dominant contribution is the t-channel accounting for about $\sigma \times \mathrm{BR}=53.5 \mathrm{pb}$, followed by the $\mathrm{Wt}$ events for $18.0 \mathrm{pb}$ [15] and by the $\mathrm{W}^{*}$ channel for $2.2 \mathrm{pb}$ [14] The corresponding cross-sections have been computed at NLO, but the present analyses make use the LO TopRex generator, normalized to NLO predictions.

The present analyses make use only of the leptonic decays $(\mathrm{e}, \mu)$ of the $\mathrm{W}$ boson, leading to a common preselection based on the presence of one high-p $\mathrm{p}_{\mathrm{T}}$ lepton above $25 \mathrm{GeV} / \mathrm{c}$, a large transverse missing energy, at least two high- $\mathrm{p}_{\mathrm{T}}$ jets above $30 \mathrm{GeV} / \mathrm{c}$, among them at least one must be tagged as a b-jet. A secondary high$\mathrm{p}_{\mathrm{T}}$ lepton veto is applied to reduce di-lepton events and a jet invariant mass cuts above the $\mathrm{Z}$ mass is used to reduce di-boson contamination.
Contrary to the top mass analysis, single-top measurements are affected by a significant level of background contamination. Top pair production constitutes the dominant source with a cross-section about 3 times as large as the total single-top, with 'lepton+jets' and 'di-lepton' channels, followed by the tau decays involving one or two top quarks. $\mathrm{Wb}, \mathrm{b} \overline{\mathrm{b}}$ and $\mathrm{Wc}, \mathrm{c} \overline{\mathrm{c}}$ events also represent a significant background. Corresponding cross-sections are based on specific calculations imposing "realistic" constraints to final state partons, and lead to $300 \mathrm{pb}$ [?] for a lepton and at least 2 b-jets above $15 \mathrm{GeV} / \mathrm{c}$.

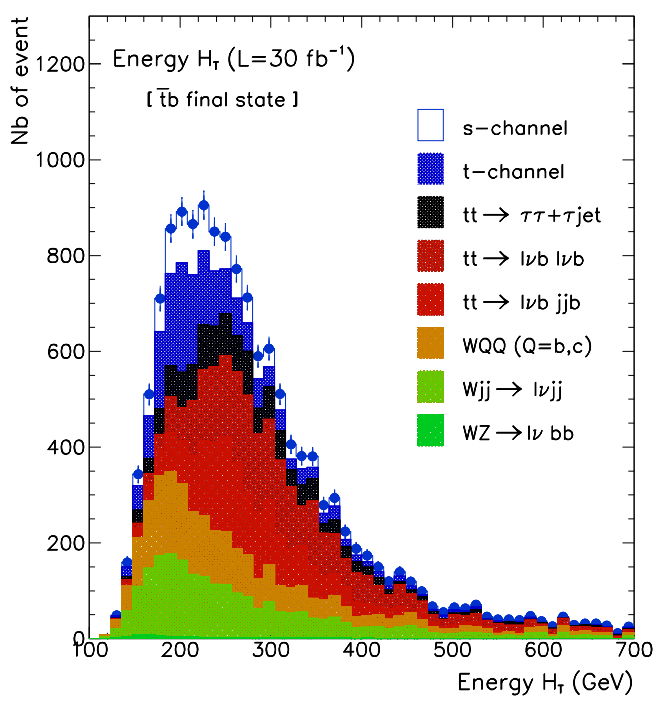

Fig. 4. Invariant mass reconstructed in the "2-btag" sample

$\mathrm{W}+$ light jets events constitute a major source of background because of a cross-section several orders of magnitude above the signal's one. This processus can mimic the signal in the case where one or two light jets are (wrongly) tagged as a b-jet(s). Total cross-sections may be found in Ref. [?] for $\mathrm{W}+\mathrm{j}, \mathrm{W}+\mathrm{jj}$ and $\mathrm{W}+\mathrm{jjj}$ events with realistic thresholds put on the lepton and jets acceptance and $\mathrm{p}_{\mathrm{T}}$. For these analyses, the Herwig generator has been used and the results normalized to NLO cross-sections when available. Di-boson events constitute a background to our signal. The dominant contribution comes from the $\mathrm{WZ} \rightarrow \mathrm{l} \nu \mathrm{bb} \overline{\mathrm{b}}$ events with a cross-section of $\sigma \times \mathrm{BR}=440 \mathrm{fb}$.

\section{$4.2 \mathrm{Wg}$ cross-section}

The selection of t-channel is largely based upon topological variables. Events with exactly two high-p $\mathrm{p}_{\mathrm{T}}$ jets are selected to reduce the top pair events contamination. Among them, exactly one jet is required to be b-tagged, the second b-jet being expected at high rapidity region outside 
the vertex tracker acceptance. The non-b tagged jet must point toward the forward rapidity region with $|\eta|>2.5$. A window is applied upon the reconstructed leptonic top mass $\mathrm{M}_{l \nu \mathrm{b}}$ to help reduce non-top events. Other requirements are applied on the total energy $\mathrm{H}_{\mathrm{T}}$ defined as the scalar sum of physics objects transverse momentum, to reduce further $\mathrm{W}+$ jets and top pair event contamination.

About 7,000 signal events are expected for $30 \mathrm{fb}^{-1}$ with a ratio $\mathrm{S} / \mathrm{B}$ of 3 . The corresponding statistical sensitivity $\sqrt{\mathrm{S}+\mathrm{B}} / \mathrm{S}$ is shown to stay below $1.5 \%$. This analysis will be dominated by systematic uncertainties, originating from the precision of the luminosity determination, and the (b)-jet energy scale.

\section{$4.3 \mathrm{~W}+\mathrm{t}$ cross-section}

The event selection requires that exactly three high $\mathrm{p}_{\mathrm{T}}$ jets are detected, in order to reduce top pair and $\mathrm{W}+$ jet contamination. Among those jets, exactly one jet must be b-tagged. The reconstructed leptonic top mass, as well as $\mathrm{H}_{\mathrm{T}}$ and the total mass of the events are used to improve background rejection. The reconstructed hadronic $\mathrm{W}$ boson mass is used as an additional constraint to further reduce non-top background.

Typical efficiencies at the $1 \%$ level are found for a ratio $\mathrm{S} / \mathrm{B} \approx 15 \%$. For $\mathcal{L}=30 \mathrm{fb}^{-1}$ this results in a statistical sensitivity of about $4 \%$.

\section{4 $\mathrm{W}^{*}$ cross-section}

The selection of the s-channel events requires exactly two high $\mathrm{p}_{\mathrm{T}}$ b-tagged jets with a veto of an extra light jet above $15 \mathrm{GeV} / \mathrm{c}$. This criterium allows to reduce significantely top pair contamination as well as $\mathrm{W}+$ light jets events. Extra requirements based on cuts on the reconstructed top mass $\mathrm{M}(\mathrm{l} \nu \mathrm{b})$ and $\mathrm{H}_{\mathrm{T}}$ are used to further purify the sample. As for the other channels, the analysis can be performed separately for the $t \bar{b}$ and $\overline{\mathrm{t}} \mathrm{b}$ final states in order to help reduce charge-symmetric backgrounds like top pairs.

About 1,200 (800) signal events are expected in the $t \bar{b}(\overline{\mathrm{t} b})$ final state, for ratio a S/B ranging between 10-15\%. A statistical sensitivity of $7-8 \%$ seems achievable for $30 \mathrm{fb}^{-1}$. As for the two previous analyses, systematics will be a major factor. In this case, on top of the sources detailed in 4.2 , the uncertainty on the background normalization (W+heavy quarks, $\mathrm{W}+$ jets and top pair) will be one of the main source of systematics.

\subsection{Sensitivity to new physics}

In 2HDM, two higgs fields are assumed to generate the electro-weak symmetry breaking. This results into five physical states associated each to a higgs boson : three neutral (h, $\mathrm{H}$ and $\mathrm{A}$ ) and two charged bosons $\left(\mathrm{H}^{+}, \mathrm{H}^{-}\right)$. In SUSY, Higgs mass spectrum is determined and depends upon one mass, usually $\mathrm{m}_{\mathrm{A}}$, and the $\tan \beta$ parameter, defined as the ratio of the two vacuum expectation values (vev) for the higgs fields.

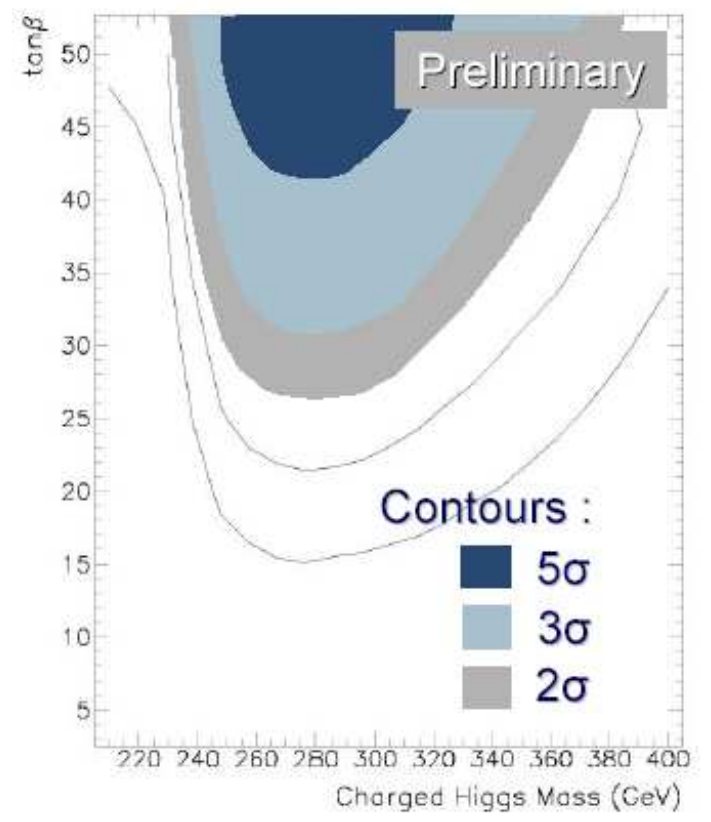

Fig. 5. Discovery contours for a charged higgs in the $\left(\mathrm{m}_{\mathrm{H}}, \tan \beta\right)$ plane, using only the s-channel cross-section measurement

In those models, the contribution to the $\mathrm{W}^{*}$ channel is expected to be enhanced due to the addition of a graph involving the $\mathrm{H}^{ \pm}$with $\mathrm{H}^{+} \rightarrow \mathrm{t} \overline{\mathrm{b}}$, resulting in a deviation from the SM expectations. The magnitude of the $\mathrm{H}^{+}$tb coupling depending upon $\mathrm{m}_{\mathrm{H}^{ \pm}}$and $\tan \beta$, the analysis has been performed as a scan in the $\left(\mathrm{m}_{\mathrm{H}}, \tan \beta\right)$ plane. Using the same selection as defined in the $\mathrm{W}^{*}$ analysis, a $5 \sigma$ discovery seems achievable for high $\tan \beta$ and higgs mass above $250 \mathrm{GeV} / \mathrm{c}^{2}$, as shown in Fig. 5. This result should be improved by using a specific selection using the specific property of the scalar $\mathrm{H}^{ \pm}$. It should also benefit from the combination with the $\mathrm{W}+\mathrm{t}$ events searches.

\section{Acknowledgments}

I would like to thank the Atlas collaboration members of the Top physics group for helpful discussions. Among them I am grateful to D. Pallin (LPCC) for his comments about the top mass measurement methods. I would like to thank also F. Hubaut and P. Pralavorio (CPPM) for fruitful discussions about the top quark properties measurements.

\section{References}

1. S. Heinemeyer, G. Weiglin, hep-ph/0307177 (2003)

2. LEP EWWG page / Summer 2005

3. R. Bonciani et al., Nucl. Phys. B 528 (1998) 424 
4. I. Borjanovic et al., hep-ex/0403021

5. V.Simak et al., ATL-COM-PHYS-99-073

6. C.S. Hill et al., hep-ex $/ 0501043$

7. D. Chakraborty et al., hep-ph/0303092

8. F.Hubaut et al. SN-ATLAS-2005-052

9. V. Simael et al., ATL-PHYS-2001-018

10. H.S. Do et al., Phys. Rev.D67(2003) 091501

11. M.Beg et al., Phys. Rev.Lett. 38(1977) 1252

12. G.L.kane et al., Phys.Rev.D45(1992)124

13. D. Chakraborty, hep-ex/0212027

14. J. Campbell et al., hep-ph/0408158

15. F. Maltoni, HCP 2005 proceedings

16. J. Campbell et al., hep-ph/0308195 